\title{
Self-assembly of metal nanowires induced by ac electric fields
}

\author{
Pablo García-Sánchez, ${ }^{1,}$ a) Juan J. Arcenegui, ${ }^{1}$ Hywel Morgan, ${ }^{2}$ and Antonio Ramos ${ }^{1}$ \\ 1) Depto. Electrónica y Electromagnetismo. Facultad de Física. Universidad de Sevilla. Avda. Reina Mercedes s/n, \\ 41012. Sevilla (Spain). \\ ${ }^{2)}$ School of Electronics and Computer Science, University of Southampton, Highfield, Southampton SO17 1BJ, \\ $U K$.
}

(Dated: 10 December 2014)

We describe the reversible assembly of an aqueous suspension of metal nanowires into two different 2-dimensional stable configurations. The assembly is induced by an AC electric field of magnitude around 10 $\mathrm{kV} / \mathrm{m}$. It is known that single metal nanowires orientate parallel to the electric field for all values of applied frequency, according to two different mechanisms depending on the frequency. These different mechanisms also govern the mutual interaction between nanowires, which leads to directed-assembly into distinctive structures, the shape of which depends on the frequency of the applied field. We show that for frequencies higher than the typical frequency for charging the electrical double layer at the metal-electrolyte interface, dipole-dipole interaction leads to the formation of chains of nanowires. For lower frequencies the nanowires form wavy bands perpendicular to the electric field direction. This behavior appears to be driven by the electroosmotic flow induced on the metal surface of the nanowires. Remarkably, no similar structures have been reported in previous studies of nanowires.

Keywords: Nanowires, Assembly, AC Electroosmosis, Induced-Charge Electroosmosis, Electro-orientation

Nanowires synthesis and manipulation ${ }^{1}$ has received considerable attention with potential applications in several fields such as biotechnology ${ }^{2}$, microelectronics ${ }^{3}$ and solar cells ${ }^{4}$. Electric fields are widely used to manipulate micro- and nano-particles ${ }^{5,6}$. For example, Smith et al. ${ }^{7}$ used electric fields to position individual gold nanowires between two electrodes. The alignment of single-wall carbon nanotubes upon application of ac fields was studied in refs. ${ }^{8,9}$. Fan et al. ${ }^{10}$ used dielectrophoretic and electrophoretic forces to align and transport metal nanowires in water solutions. Golovin et al. ${ }^{11}$ reported electrically reconfigurable optical metamaterials based on nanorod manipulation in dielectric liquids. The dielectrophoretic assembly of metal nanowires networks and $\mathrm{ZnO}$ nanorods have also been studied ${ }^{12,13}$. The electro-rotation and electro-orientation of single silver nanowires in aqueous suspensions has also been studied, both experimentally and from the fundamental theoretical perspective ${ }^{14,15}$. The behavior of a metal nanowire is driven by the interaction of the electric field with the induced charge in the electrical double layer at the metal-electrolyte interface. In this letter we study the ensemble behavior of $\mathrm{Ag}$ nanowires in $\mathrm{KCl}$ electrolytes. The nanowires are much heavier than water and therefore sink to the bottom of the device, where they undergo strong Brownian motion. This manifests itself as a continuous random change in their orientation. Thus, the nanowires freely rotate when subjected to an external electric field.

Nanowire assembly was investigated using a fluidic channel (1 cm long) consisting of a shallow PDMS

\footnotetext{
a) Electronic mail: corresponding author: pablogarcia@us.es
}

chamber (500 $\mu \mathrm{m}$ deep) bonded to a glass substrate. Two electrodes made of aluminum foil are placed at either end of this. Thus, the electrode gap is $1 \mathrm{~cm}$, see Figure 1A. The chamber was filled with a $\mathrm{KCl}$ aqueous solution of a given conductivity and a tiny drop of a concentrated nanowire dispersion injected at the center of the electrode gap. In this way, the nanowires are far from the electrodes and any shortcircuit when nanowire
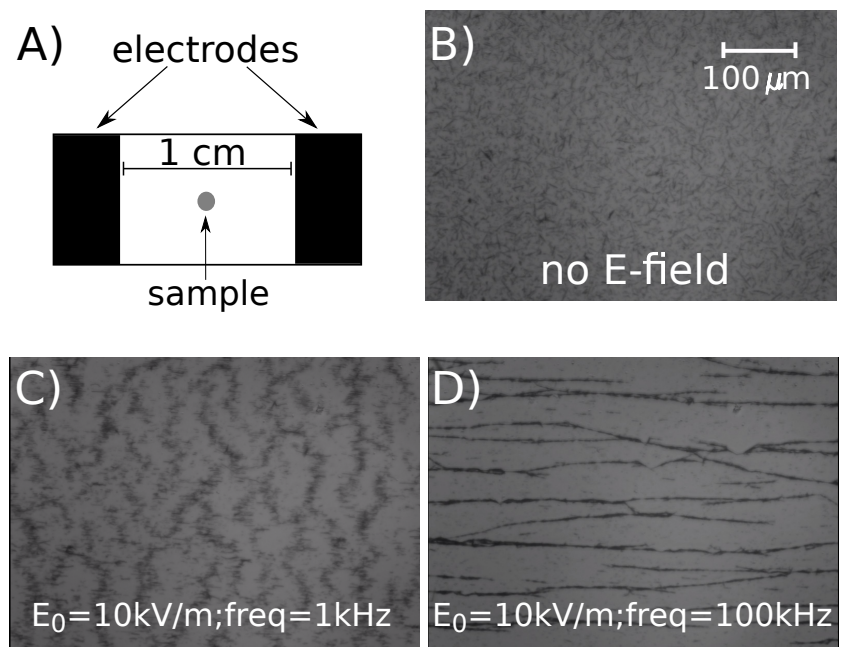

FIG. 1. A)Diagram showing the experimental system consisting of two thin film metal electrodes at either end of a shallow microfluidic structure made in PDMS. A sample of the Ag nanowire suspension is injected into the electrolyte between the two electrodes. B)Prior to application of the electric field, nanowires are randomly oriented and distributed. C)For an AC electric field of $1 \mathrm{kHz}$, nanowires stack, forming bands. D)For an AC electric field of $100 \mathrm{kHz}$, nanowires form chains parallel with the field. See supplementary material for a video of the nanowire assembly ${ }^{16}$. 
chains are formed is avoided. Experiments could be run for tens of minutes or even hours before Brownian motion brought the nanowires close to the electrodes. Also, fluid flow and bubbles or electrochemical products generated at the electrodes are far from nanowires and do not affect their behavior. Samples were prepared from silver nanowires supplied by "Nanostructured and Amorphous Materials Inc." as a paste consisting of $70 \%$ wt silver and $30 \%$ wt ethanol. The wires are cylindrical with a dispersion in length and width. SEM images were used to measure the nanowire lengths, which ranged between 2 and 8 microns and widths between 160 and $320 \mathrm{~nm}$. Prior to injection of the sample, the nanowires were washed several times in $\mathrm{KCl}$ solutions with the same conductivity as the electrolyte in the fluidic chamber. PVP (polyvinylpyrrolidone) is added during the synthesis of the silver nanowires and might be present on the silver surface, but this is likely to be in small amounts after extensive washing. A voltage generator and amplifier was used to apply AC signals to the electrodes with an amplitude of 100 $\mathrm{V}_{\mathrm{pp}}$ and frequencies ranging from $100 \mathrm{~Hz}$ to $1 \mathrm{MHz}$. The behavior of the nanowires was observed with an inverted microscope and, for all frequencies of $\mathrm{AC}$ field, the wires align with the electric field direction, as expected from previous observations of the behavior of single nanowires ${ }^{15}$. As shown in ref. ${ }^{15}$, the alignment is stronger at high frequencies and is due to the torque on the induced dipole while at low frequency the alignment is due to induced-charge electroosmotic (ICEO) flow around the particle. However, the interaction between many nanowires gives rise to distinctive $2 \mathrm{D}$ structures depending on the frequency of the applied field.

Figure 1B shows a picture of the suspension before application of the $\mathrm{AC}$ field, the orientation of the nanowires is random and no patterns are observed. Upon application of a low frequency AC signal $(1 \mathrm{kHz})$, the nanowires stack, forming bands orthogonal to the electric field and with a wavy shape, see Figure $1 \mathrm{C}$. Remarkably, no similar structures have been reported in previous studies of nanowires. However, for an $\mathrm{AC}$ signal with a high frequency $(100 \mathrm{kHz})$, the nanowires form chains, see Figure 1D. Both structures are stable as long as the electric field is applied and they have been observed for nanowires dispersed in DI water and in $\mathrm{KCl}$ solutions with conductivities up to $15 \mathrm{mS} / \mathrm{m}$, although for this conductivity the stacking took longer to develop. Experiments for higher liquid conductivities were not posible because the nanowires stick to each other and to the bottom glass (presumable due to DLVO forces from collapse of the electrical double layer). The assembly dynamics of the two states can be observed on the video in the supplementary material for an electrolyte conductivity of $1.5 \mathrm{mS} / \mathrm{m}$. It is also observed that, after the electric field is switched off, Brownian forces redisperse the nanowires and the initial state is recovered after a few minutes. This demonstrates that

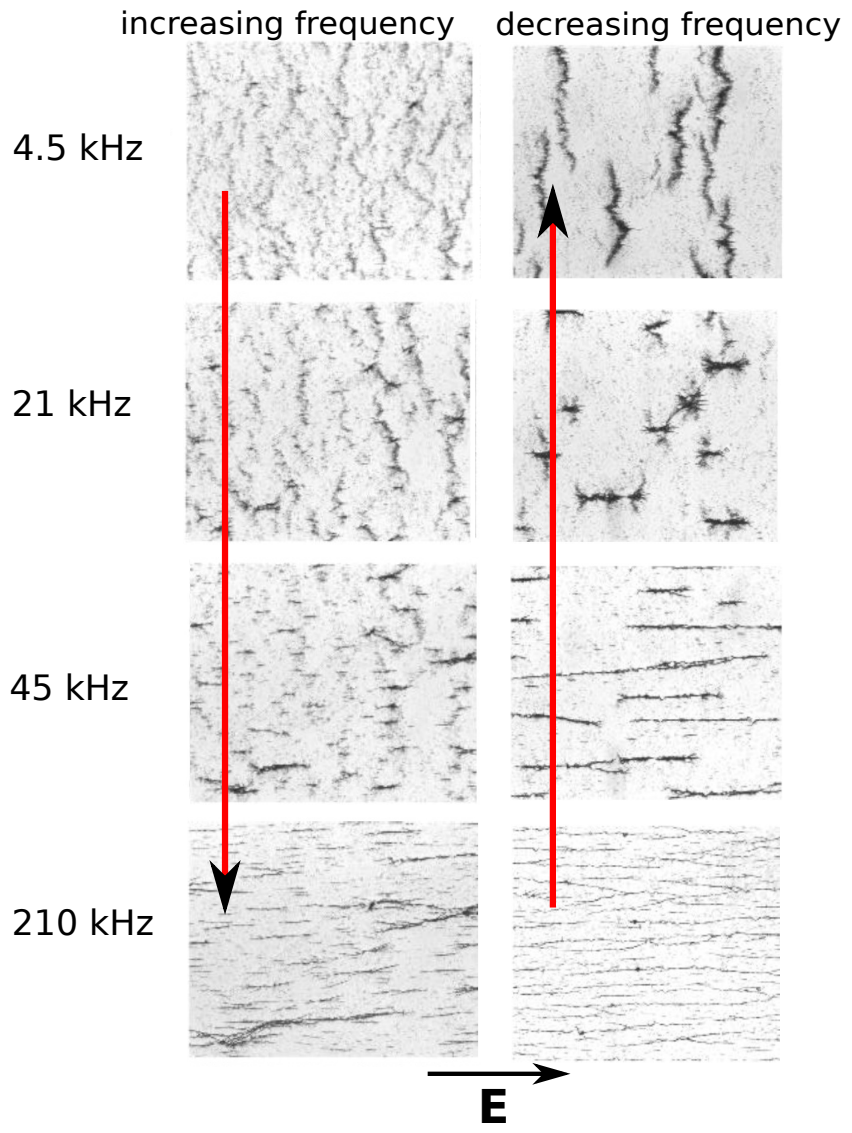

FIG. 2. Microscope images of the metal nanowire networks for several frequencies of the applied electric field. All images are captured in steady state, i.e. several minutes after application of the signal. The amplitude of the electric field in these experiments is $E_{0}=10 \mathrm{kV} / \mathrm{m}$ and the electrolyte conductivity is $1.5 \mathrm{mS} / \mathrm{m}$. (Left column) Scanning from low to high frequency. (Right column) Scanning from high frequency to low.

the assembly is reversible.

Figure 2 shows images of the nanowire networks for intermediate frequencies of the applied field. In this case, the signal frequency was changed quasistatically. For these intermediate frequencies, the assembly shows a competition between the two main mechanisms and the patterns depend on history. The left column in figure 2 shows the results of a process starting with a low frequency of the applied field. As the frequency is increased, some complicated networks are formed and, finally, the chain state is reproduced if the frequency is high enough (for simplicity, images are shown only for four frequencies values). Right column in figure 2 shows images of the same process but starting with a high frequency. In this case, as the frequency is decreased, complicated nanowire networks also appear, but they are clearly different. Again, stacking of the nanowires is found if the frequency is low enough. 
In order to explore if the collective behavior of many nanowires can be understood from the interaction between two nanowires, experiments were performed with a low concentration of nanowire (ideally, only two nanowires). Video 2 (supplementary material) ${ }^{17}$ shows that at high frequencies $(600 \mathrm{kHz})$ particles order by attaching end to end, forming a short chain. When the nanowire concentration is high this extends to very long chains. As the applied frequency decreases,neighboring particles remain aligned with the field but move until they are side by side. This relative arrangement (stacking) gives rise to the bands observed when the concentration of nanowires is higher. The conductivity for the experiments shown in video 2 was $1.5 \mathrm{mS} / \mathrm{m}$; similar experiments were repeated the observations for 5 and $15 \mathrm{mS} / \mathrm{m}$. In all cases the same relative motion was observed between neighboring nanowires. The videos were further analysed to determine the transition frequencies between the chain and intermediate states, as well as the transition between the intermediate state and stacking. The continuous lines shown in Figure 3 correspond to these transition frequencies, plotted as a function of electrolyte conductivity. The upper continuous line is the transition from chaining to intermediate state. Thus, the region above that line corresponds to the experimental conditions where chains form. The lower continuous line in Figure 3 is the transition from an intermediate state to stacking, and the region below that line corresponds to experimental conditions where stacking is found.

An explanation for this frequency behavior is found in the analysis of the mechanism of nanowire polarization. In refs. ${ }^{14,15}$, we showed that the electrical behavior of a metal nanowire suspended in an electrolyte is described by the charging of the electrical double layer (EDL) induced at the metal-electrolyte interface. The

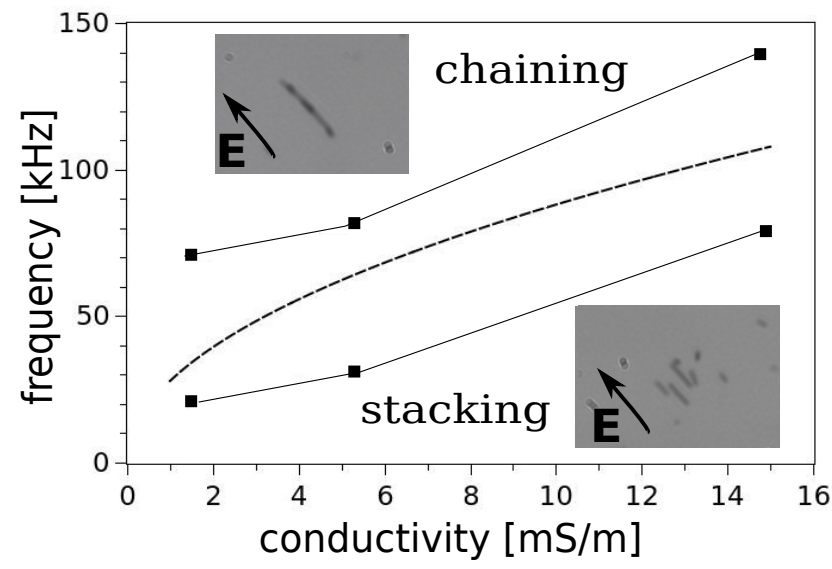

FIG. 3. Frequency-conductivity map showing the regions where each configuration was found. The dashed line corresponds to the frequency obtained from equation (1).
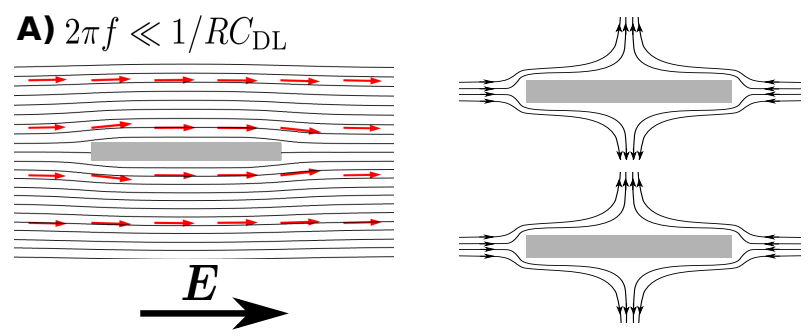

B) $2 \pi f \gg 1 / R C_{\mathrm{DL}}$
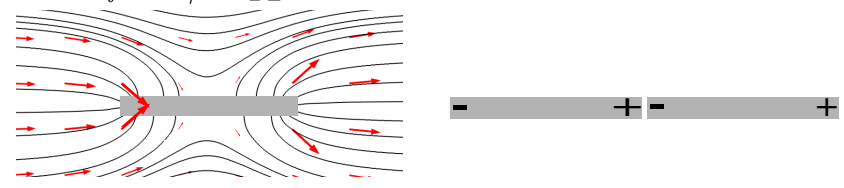

FIG. 4. A) (left) Electric field lines around a metal cylinder for a low frequency AC field. (right) Induced-charge electroosmosis is generated on the metal nanowire surfaces, driving a hydrodynamic interaction between neighboring nanowires. B)(left) Electric field lines for high frequency $\mathrm{AC}$ fields. The electric field is strongly perturbed by the metal particle. (right) Dipole-dipole interactions lead to particle chainning.

current coming from the electrolyte bulk brings ions to this interface, where they accumulate to form the EDL. This charging process has a typical time similar to the $R C$-time for charging a capacitor, $C$, with the current through a resistor, $R$. In this case, $C$ is given by the EDL capacitance and $R$ by the resistance of the electrolyte bulk. Electrorotation and electroorientation experiments of metal nanowires are in accordance with this polarization mechanism. For angular frequencies of the AC signal much lower than the reciprocal of the typical time $(\omega \ll 1 / R C)$, the EDL is fully charged and the electric field lines go around the nanowire. For the case of a slender cylinder, the electric field is only slightly perturbed, see Figure 4A. However, for angular frequencies much higher than $1 / R C$, there is no time to charge the EDL and the electric field lines intersect perpendicularly with the metal surface, see Figure 4B. In this case the electric field is strongly perturbed by the particle and, consequently, the induced dipole in the particle becomes important. Particle Chaining corresponds to the expected behavior arising from dipole-dipole interaction ${ }^{5}$ and is in accordance with previous observations of metal nanoparticles described in ref. ${ }^{18}$. However, dipole-dipole interaction at low frequencies can be neglected and the origin of the observations in this case can be explained, at least in part, by induced-charge electroosmosis (ICEO) generated on the metal nanowire surfaces, as first described theoretically by Saintillan et al. ${ }^{19}$. Rose et $a l^{20}{ }^{20}$ observed that the hydrodynamic interaction between a pair of metal nanowires was caused by ICEO; when two nanowires approach, they align end-to-end, slide next to one another and then move apart from 
their centers. This is the relative motion expected from the flow field calculated in ref. ${ }^{19}$, see figure $4 \mathrm{~A}$ (right). Also, it is apparent from our experiments with few nanowires (Fig. 3) that after two nanowires move apart, they remain within an equilibrium distance of a few microns. The reason for this is unclear but might be due to some electrical interaction not caused by ICEO flow. According to Saintillan et al. ${ }^{19}$, the pair hydrodynamic interaction between metal nanowires due to ICEO is such that there is attraction for angles near the poles and repulsion near the equator. There is a neutral line situatued more or less at $45^{0}$. This may be the reason that in experiments the most frequent relative position between two nanowires is side by side with their centers approximately along this line. This could also be the mechanism that creates the wavy bands seen in figures 1 and 2.

As mentioned above, our silver nanowires have a dipersion in length and width. The average length is around $6.5 \mu \mathrm{m}$ and their aspect ratio is around 0.04 . We have numerically computed the typical angular frequency for charging the EDL of a metal cylinder with this aspect ratio, given by the following expression:

$$
\omega=2 \pi f=10.97 \frac{\sigma}{a C_{D L}}
$$

where $2 a$ is the nanowire length, $C_{D L}$ is the EDL specific capacitance and $\sigma$ is the electrolyte conductivity. It can be assumed that $C_{D L}$ is given by the Debye-Hückel approximation ${ }^{21}, C_{D L}=\varepsilon / \lambda_{D}$, where $\varepsilon$ is the electrolyte permittivity and $\lambda_{D}$ the Debye length, i.e. the thickness of the EDL. Electrorotation and electroorientation results in refs. ${ }^{14,15}$ validate this assumption. Therefore the relaxation frequency can be calculated, shown by the dashed line in figure 3 for a nanowire with length $2 a=6.5 \mu \mathrm{m}$ plotted as a function of electrolyte conductivity. Chaining is clearly observed for frequencies higher than typical for charging the EDL, while stacking is clearly observed for frequencies lower than this, as expected from our hypothesis. In the light of these mechanisms, the hysteresis shown in figure 2 can be explained as follows. When decreasing the frequency quasistatically, the chains break into segments composed of many nanowires. These segments behave as bigger wires. At low frequency they show the pair hydrodynamic interaction corresponding to larger slender objects and form wavy bands which are thicker than when starting from isolated nanowires.

In conclusion we have described the reversible selfassembly of metal nanowires in aqueous suspensions induced by $\mathrm{AC}$ electric fields. For frequencies of the AC signals above the inverse charge relaxation time of the EDL, the particle-particle interactions lead to nanowire chaining, while for low frequencies of the signal stacking of the particles is observed. This frequencydependent behavior has been characterised as a function of electrolyte conductivity and based on our previous observations of the $\mathrm{AC}$ electrokinetics of single metal nanowires, the mechanisms responsible for the assembly were identified as dipole-dipole interaction for chaining and ICEO flow on the metal surface for stacking. Finally, the development of electric-field based methods for alignment and directed assembly of nanowires, together with a further understanding of these mechanisms could find application in many areas of nanotechnology.

\section{ACKNOWLEDGMENTS}

We acknowledge financial support from Junta de Andalucía and Spanish Government Ministry MEC under contracts, respectively, P09-FQM-4584 and FIS201125161. HM acknowledges funding from the Royal Society

${ }^{1} \mathrm{G}$. Cao, Synthesis, properties and applications (World Scientific, 2004).

${ }^{2}$ Y. Cui, Q. Wei, H. Park, and C. M. Lieber, Science 293, 1289 (2001).

${ }^{3}$ Y. Li, F. Qian, J. Xiang, and C. M. Lieber, Materials Today 9, 18 (2006).

${ }^{4}$ M. Law, L. E. Greene, J. C. Johnson, R. Saykally, and P. Yang, Nature Materials 4, 455 (2005).

${ }^{5}$ T. B. Jones, Electromechanics of Particles (Cambridge University Press, 1995).

${ }^{6} \mathrm{H}$. Morgan and N. G. Green, AC Electrokinetics: colloids and nanoparticles. (Research Studies Press Ltd., 2003).

${ }^{7}$ P. A. Smith, C. D. Nordquist, T. N. Jackson, T. S. Mayer, B. R. Martin, J. Mbindyo, and T. E. Mallouk, Applied Physics Letters 77, 1399 (2000).

${ }^{8}$ X. Chen, T. Saito, H. Yamada, and K. Matsushige, Applied Physics letters 78, 3714 (2001).

${ }^{9} \mathrm{~J}$. Li, Q. Zhang, N. Peng, and Q. Zhu, Applied Physics Letters 86, 153116 (2005).

${ }^{10}$ D. Fan, R. Cammarata, and C. Chien, Applied Physics Letters 92, 093115 (2008).

${ }^{11}$ A. B. Golovin and O. D. Lavrentovich, Applied Physics Letters 95, 254104 (2009).

${ }^{12}$ S. J. Papadakis, Z. Gu, and D. H. Gracias, Applied Physics Letters 88, 233118 (2006).

${ }^{13}$ D. Wang, R. Zhu, Z. Zhou, and X. Ye, Applied Physics Letters 90, 103110 (2007).

${ }^{14}$ J. J. Arcenegui, P. García-Sánchez, H. Morgan, and A. Ramos, Physical Review E 88, 033025 (2013).

${ }^{15}$ J. J. Arcenegui, P. García-Sánchez, H. Morgan, and A. Ramos, Physical Review E 88, 063018 (2013).

${ }^{16}$ See supplementary material at [URL will be inserted by AIP] for a video of the nanowire assembly.

${ }^{17}$ See supplementary material at [URL will be inserted by AIP] for a video of the nanowires relative motion..

${ }^{18}$ K. D. Hermanson, S. O. Lumsdon, J. P. Williams, E. W. Kaler, and O. D. Velev, Science 294, 1082 (2001).

${ }^{19}$ D. Saintillan, E. Darvel, and E. Shaqfeh, Journal of Fluid Mechanics 563, 223 (2006).

${ }^{20}$ K. A. Rose, B. Hoffman, D. Saintillan, E. S. Shaqfeh, and J. G. Santiago, Physical Review E 79, 011402 (2009).

${ }^{21} \mathrm{R}$. Hunter, Introduction to Modern Colloid Science (Oxford University Press, 1993). 